Original article

\title{
Cannabis use and suicide attempts among 86,254 adolescents aged 12-15 years from 21 low- and middle-income countries
}

\author{
Andre F. Carvalho ${ }^{\mathrm{a}, \mathrm{b}, *}$, Brendon Stubbs $^{\mathrm{c}, \mathrm{d}, \mathrm{e}}$, Davy Vancampfortt, ${ }^{\mathrm{f}, \mathrm{g}}$, Stefan Kloiber ${ }^{\mathrm{a}, \mathrm{b}}$, \\ Michael Maes $^{\mathrm{h}, \mathrm{i}}$, Joseph Firth ${ }^{\mathrm{j}, \mathrm{k}, \mathrm{l}}$, Paul A. Kurdyak ${ }^{\mathrm{m}, \mathrm{n}, \mathrm{o}}$, Dan J. Stein ${ }^{\mathrm{p}}$, Jürgen Rehm,q,r,s \\ Ai Koyanagi ${ }^{\mathrm{t}}$
}

a Department of Psychiatry, University of Toronto, Toronto, ON, Canada

${ }^{\mathrm{b}}$ Centre for Addiction and Mental Health $(\mathrm{CAMH})$, Toronto, ON, Canada

' South London and Maudsley NHS Foundation Trust, Denmark Hill, London, SE5 8AZ, UK

d Institute of Psychiatry, Psychology and Neuroscience (IoPPN), King's College London, De Crespigny Park, London, AF, SE5 8, UK

e Faculty of Health, Social Care and Education, Anglia Ruskin University, Chelmsford, CM1 1SQ UK

${ }^{\mathrm{f}} \mathrm{KU}$ Leuven Department of Rehabilitation Sciences, Leuven, Belgium

${ }^{\mathrm{g}} \mathrm{KU}$ Leuven Department of Neurosciences, UPC KU Leuven, Kortenberg, Belgium

${ }^{\mathrm{h}}$ IMPACT Strategic Research Centre, School of Medicine, Barwon Health, Deakin University, Geelong, Victoria, Australia

i Department of Psychiatry, Faculty of Medicine, Chulalongkorn University, Bangkok, Thailand

${ }^{\mathbf{j}}$ NICM Health Research Institute, School of Science and Health, Western Sydney University, Sydney, Australia

${ }^{\mathrm{k}}$ Division of Psychology and Mental Health, Faculty of Biology, Medicine and Health, University of Manchester, Manchester, UK

${ }^{1}$ Centre for Youth Mental Health, University of Melbourne, Melbourne, Australia

${ }^{\mathrm{m}}$ Health Outcomes and Performance Evaluation (HOPE) Research Unit within the Social and Epidemiological Research Program, Centre for Addiction and Mental Health, Toronto, ON, Canada

${ }^{\mathrm{n}}$ Department of Psychiatry and Institute of Mental Health Policy Research, University of Toronto, Toronto, Ontario, Canada

${ }^{\circ}$ Mental Health and Addictions Research Program at the Institute for Clinical Evaluative Science (ICES), Toronto, ON, Canada

P Department of Psychiatry and MRC Unit on Risk and Resilience in Mental Disorders, Faculty of Health Sciences, University of Cape Town and Groote Schuur

Hospital, Cape Town, South Africa

${ }^{\mathbf{q}}$ Institute for Clinical Psychology and Psychotherapy, Technische Universität Dresden, Chemnitzer Str. 46, 01187, Dresden, Germany

${ }^{\mathrm{r}}$ Addiction Policy, Dalla Lana School of Public Health, University of Toronto, 155 College Street, Toronto, ON, M5T 3M7, Canada

${ }^{\mathrm{s}}$ Institute of Medical Science, University of Toronto, Faculty of Medicine, Medical Sciences Building, 1 King's College Circle, Toronto, ON, M5S 1A8, Canada

${ }^{\mathrm{t}}$ Research and Development Unit, Parc Sanitari Sant Joan de Déu/CIBERSAM, Universitat de Barcelona, Fundació Sant Joan de Déu, Dr Antoni Pujadas, 42,

Sant Boi de Llobregat, Barcelona, 08830, Spain

\section{A R T I C L E I N F O}

\section{Article history:}

Received 25 August 2018

Received in revised form 17 October 2018

Accepted 18 October 2018

Available online 15 November 2018

\section{Keywords:}

Cannabis

Survey

Suicide

Adolescence

Psychiatry

Epidemiology

\begin{abstract}
A B S T R A C T
Background: Evidence suggests that cannabis use may be associated with suicidality in adolescence. Nevertheless, very few studies have assessed this association in low- and middle-income countries (LMICs). In this cross-sectional survey, we investigated the association of cannabis use and suicidal attempts in adolescents from 21 LMICs, adjusting for potential confounders.

Method: Data from the Global school-based Student Health Survey was analyzed in 86,254 adolescents from 21 countries [mean (SD) age $=13.7$ ( 0.9 ) years; $49.0 \%$ girls]. Suicide attempts during past year and cannabis during past month and lifetime were assessed. Multivariable logistic regression analyses were conducted.

Results: The overall prevalence of past 30-day cannabis use was $2.8 \%$ and the age-sex adjusted prevalence varied from $0.5 \%$ (Laos) to $37.6 \%$ (Samoa), while the overall prevalence of lifetime cannabis use was $3.9 \%$ (range $0.5 \%-44.9 \%$ ). The overall prevalence of suicide attempts during the past year was $10.5 \%$. Following multivariable adjustment to potential confounding variables, past 30-day cannabis use was significantly associated with suicide attempts $(\mathrm{OR}=2.03 ; 95 \% \mathrm{CI}$ : $1.42-2.91)$. Lifetime cannabis use was also independently associated with suicide attempts ( $\mathrm{OR}=2.30 ; 95 \% \mathrm{CI}: 1.74-3.04)$.

Conclusion: Our data indicate that cannabis use is associated with a greater likelihood for suicide attempts in adolescents living in LMICs. The causality of this association should be confirmed/refuted in prospective studies to further inform public health policies for suicide prevention in LMICs.
\end{abstract}

(C) 2018 Elsevier Masson SAS. All rights reserved. 


\section{Introduction}

The putative association between cannabis use and suicidality has been a matter of debate in the literature. For example, the last report from the Institute for Health Metrics Science and Evaluation (IHME) group on the burden of mental and substance use disorders on deaths by suicide did not include cannabis use as a risk factor for suicide [1]. In addition, a recent review and meta-analysis found a lack of consistent evidence for an association between acute cannabis use and imminent suicidality [2]. Although an association between chronic cannabis use and suicidality was suggested, the credibility of the evidence was limited by heterogeneous definitions of exposure (e.g. any cannabis use, cannabis use disorder, chronic cannabis use, etc.) and outcome (i.e. suicidal ideation, suicide attempts and deaths due to suicide) variables across included studies [2]. Moreover, the proper controls for potential confounders which are known to be associated with suicide (e.g. environmental risk factors, mood disorders, other substance use disorders) were inconsistent across studies [2].

Another major gap in the literature is the paucity of studies that have assessed this association in samples derived from low- and middle-income countries (LMICs) [2]. This association may differ in magnitude in LMICs compared to high-income countries due at least in part to a complex interaction with relevant sociodemographic and environmental risk factors [3]. For example, poverty may comprise a particularly relevant risk factor for suicide across LMICs [4]. Accordingly, the need to develop specific preventative public health policy interventions for suicide in LMICs is an unmet priority [5].

Furthermore, a large body of evidence has suggested that cannabis use during adolescence may be particularly detrimental to brain development and to the emergence of psychopathology across the lifespan [6-8]. It has also been suggested that cannabis use during adolescence may increase the risk of suicidality during both adolescence $[2,9,10]$ and adulthood [11,12].

Given these aforementioned gaps and inconsistencies in the literature, we set out to examine the patterns of associations between cannabis use and suicide attempts in a multinational sample of adolescents living in LMICs. We hypothesized that cannabis use could be associated with suicide attempts in this population, and that this association would be robust after multivariable adjustment for potential confounders.

\section{Materials and methods}

\subsection{The survey}

Publicly available data from the Global School-Based Student Health Survey (GSHS) were analyzed. Details on this survey can be found at http://www.who.int/chp/gshs and http://www.cdc.gov/ gshs. Briefly, the GSHS was jointly developed by the WHO and the US Centers for Disease Control and Prevention (CDC), and other UN allies. The core aim of this survey was to assess and quantify risk and protective factors of major non-communicable diseases. The survey draws content from the CDC Youth Risk Behavior Survey (YRBS) for which test-retest reliability has been established [13]. The survey used a standardized two-stage probability sampling design for the selection process within each participating country. For the first stage, schools were selected with probability proportional to size sampling. The second stage involved the random selection of classrooms which included students aged 1315 years within each selected school. All students in the selected classrooms were eligible to participate in the survey regardless of age. Data collection was performed during one regular class period. The questionnaire was translated into the local language in each country and comprised multiple choice response options; students recorded their response on computer scannable sheets. All GSHS surveys were approved, in each country, by both a national government administration (most often the Ministry of Health or Education) and an institutional review board or ethics committee. Student privacy was protected through anonymous and voluntary participation, and informed consent was obtained as appropriate from the students, parents and/or school officials. Data were weighted for non-response and probability selection.

From all publicly available data, we selected all nationally representative datasets that included all the variables included in this study. If there were more than two datasets from the same country, we chose the most recent dataset. Data from 21 countries were included in the current study. The characteristics of each country or survey including the response rates are provided in Table 1. For the included countries, the survey was conducted between 2009 and 2015, and consisted of 4 low-income, 11 lower middle-income, and 6 upper middle-income countries. Lowincome countries were defined as those with an annual per capita gross national income (GNI) of 995 USD or less in 2017; lowermiddle income countries are those with a GNI per capita between 996 and 3895 USD; and upper-middle income countries were those with an annual per capita GNI between 3896 and 12,055 USD. Those definitions were based on standard parameters from the World Bank.

\subsection{Variables}

\subsubsection{Suicide attempt (dependent variable)}

Suicide attempt was assessed by the question "During the past 12 months, how many times did you actually attempt suicide?" and was defined as at least one suicide attempt in the past 12 months.

\subsubsection{Cannabis use (independent variable)}

Past 30-day and lifetime cannabis use was assessed with the question "During the past 30 days (or during your life), how many times have you used marijuana?" Country-specific slang terms for marijuana were also included in the question. Marijuana use was defined as having used marijuana on at least one day in the past 30 days (past 30-day cannabis use) or ever having used marijuana (lifetime cannabis use).

\subsubsection{Control variables}

The selection of control variables was based on past literature [2] and included age, sex, food insecurity, alcohol consumption, amphetamine use, smoking and anxiety-induced insomnia. Food insecurity was used as a proxy for socioeconomic status as there were no variables on socioeconomic status in the GSHS. Also, anxietyinduced insomnia was considered a proxy of psychiatric disorders as there were no variables on psychiatric disorders including depression in the dataset. Food insecurity was assessed by the question "During the past 30 days, how often did you go hungry because there was not enough food in your home?" Answer options were categorized as 'never', 'rarely/sometimes', and 'most of the time/always'. Alcohol consumption was defined as having had one drink containing alcohol for at least one day in the past 30 days. Amphetamine use was defined as having used amphetamines (metamphetamines) at least once in life. Smoking was defined as having smoked at least on one day during the past 30 days. Anxiety-induced insomnia was defined as replying 'most of the time' or 'always' to the question "During the past 12 months, how often have you been so worried about something that you could not sleep at night?"

\subsection{Statistical analysis}

Statistical analyses were performed with Stata 14.1 (Stata Corp LP, College station, Texas). The analysis was restricted to those aged 
Table 1

Country and survey characteristics.

\begin{tabular}{|c|c|c|c|c|c|c|}
\hline \multirow[b]{2}{*}{ Country } & \multirow[b]{2}{*}{ Year } & \multirow[b]{2}{*}{ Response rate $(\%)^{\mathrm{a}}$} & \multirow[b]{2}{*}{ Country income $^{\mathrm{b}}$} & \multirow[b]{2}{*}{$\mathrm{N}^{\mathrm{c}}$} & \multicolumn{2}{|c|}{ Cannabis use (\%) } \\
\hline & & & & & Past 30 days & Lifetime \\
\hline Argentina & 2012 & 71 & UM & 21,528 & 5.5 & 7.9 \\
\hline Bangladesh & 2014 & 91 & LM & 2753 & 1.1 & 1.2 \\
\hline Benin & 2009 & 90 & $\mathrm{~L}$ & 1170 & 2.3 & 2.7 \\
\hline Bolivia & 2012 & 88 & LM & 2804 & 2.6 & 3.5 \\
\hline Cambodia & 2013 & 85 & $\mathrm{~L}$ & 1812 & 1.3 & 1.5 \\
\hline East Timor & 2015 & 79 & LM & 1631 & 5.8 & 8.1 \\
\hline Ghana & 2012 & 82 & LM & 1110 & 7.3 & 9.3 \\
\hline Indonesia & 2015 & 94 & LM & 8806 & 1.1 & 1.7 \\
\hline Kiribati & 2011 & 85 & LM & 1340 & 5.4 & 6.2 \\
\hline Laos & 2015 & 70 & LM & 1644 & 0.5 & 0.5 \\
\hline Malaysia & 2012 & 89 & UM & 16,273 & 1.2 & 1.5 \\
\hline Mongolia & 2013 & 88 & LM & 3707 & 1.0 & 1.5 \\
\hline Mozambique & 2015 & 80 & $\mathrm{~L}$ & 668 & 1.3 & 1.7 \\
\hline Namibia & 2013 & 89 & UM & 1936 & 4.4 & 5.9 \\
\hline Peru & 2010 & 85 & UM & 2359 & 2.8 & 3.6 \\
\hline Philippines & 2015 & 79 & LM & 6162 & 5.1 & 7.1 \\
\hline Samoa & 2011 & 79 & LM & 2200 & 37.6 & 44.9 \\
\hline Solomon Islands & 2011 & 85 & LM & 925 & 11.3 & 13.6 \\
\hline Tanzania & 2014 & 87 & $\mathrm{~L}$ & 2615 & 2.1 & 3.1 \\
\hline Thailand & 2015 & 89 & UM & 4132 & 5.4 & 8.1 \\
\hline Tuvalu & 2013 & 90 & UM & 679 & 4.1 & 6.2 \\
\hline
\end{tabular}

Abbreviation: L Low income; LM Lower middle income; UM Upper middle income; H High income.

a Response rate was calculated as school response rate multiplied by student response rate.

b Country income level was based on the World Bank classification at the year of the survey in the respective countries.

c Based on sample aged 12-15 years.

d Age- and sex-adjusted.

12-15 years. Age-sex adjusted prevalence of cannabis use by country were calculated using the proportions derived from the overall sample as the standard population. We used multivariable logistic regression analysis to estimate the association between past 30-day and lifetime cannabis use (independent variable) and suicide attempt (dependent variable). We constructed six models to assess the influence of the inclusion of different variables on the association between cannabis use and suicide attempts. Specifically, a base model which adjusted only for sociodemographic factors (age, sex, food insecurity, country) was constructed. Subsequently, we added alcohol consumption, amphetamine use, smoking, and anxiety-induced insomnia individually in the base model. Finally, we adjusted for all the above-mentioned variables (fully adjusted model). Adjustment for country was done using fixed effects models as in a previous GSHS study [14]. We also tested for interaction by sex in terms of the association between cannabis consumption and suicide attempt by including the product term "sex X cannabis use" in the fully adjusted model. All variables were included in the regression analysis as categorical variables with the exception of age (continuous variable). Sampling weights and the clustered sampling design of the surveys were taken into account to obtain nationally representative estimates. Results from the logistic regression analyses are presented as odds ratios (ORs) with 95\% confidence intervals (CIs). The level of statistical significance was set at $\mathrm{p}<0.05$.

\section{Results}

The final sample consisted of 86,254 adolescents aged $12-15$ years with a mean (SD) age of 13.7 (0.9) years and 49.0\% were females. The overall age- and sex- adjusted prevalence of past-30 day and lifetime cannabis use were $2.8 \%$ [range $0.5 \%$ (Laos) to $37.6 \%$ (Samoa)] and 3.9\% [range $0.5 \%$ (Laos) to $44.9 \%$ (Samoa)], respectively (Tables 1 and 2,Fig. 1). The overall prevalence of suicide attempts was $10.1 \%$. The association between cannabis use and suicide attempt estimated by multivariable logistic regression is shown in Table 3 . In the model adjusted only for sociodemographic factors (i.e., age, sex, food insecurity, country), past 30-day cannabis use was associated with a 9.64 (95\% CI=7.36-12.61) times higher odds for suicide attempts. The inclusion of alcohol

Table 2

Sample characteristics.

\begin{tabular}{|c|c|c|c|}
\hline Characteristic & Category & Unweighted $\mathrm{N}$ & Weighted \% \\
\hline Suicide attempt (past 12 months) & Yes & 11,560 & 10.1 \\
\hline Cannabis use (past 30 days) & Yes & 3563 & 2.8 \\
\hline Cannabis use (lifetime) & Yes & 4813 & 3.9 \\
\hline \multirow[t]{4}{*}{ Age (years) } & 12 & 6062 & 12.4 \\
\hline & 13 & 22,536 & 27.9 \\
\hline & 14 & 28,996 & 32.9 \\
\hline & 15 & 28,660 & 26.7 \\
\hline Sex & Female & 45,463 & 49.0 \\
\hline Hunger due to lack of food at home & Never & 41,018 & 45.0 \\
\hline \multirow[t]{2}{*}{ (past 30 days) } & Rarely/sometimes & 39,016 & 48.2 \\
\hline & Most of the time/always & 5516 & 6.8 \\
\hline Alcohol consumption (past 30 days) & Yes & 17,118 & 10.0 \\
\hline Amphetamine use (lifetime) & Yes & 2534 & 2.7 \\
\hline Smoking (past 30 days) & Yes & 10,664 & 9.6 \\
\hline Anxiety-induced insomnia (past 12 months) & Yes & 6602 & 6.5 \\
\hline
\end{tabular}


(a) Past 30-day cannabis use

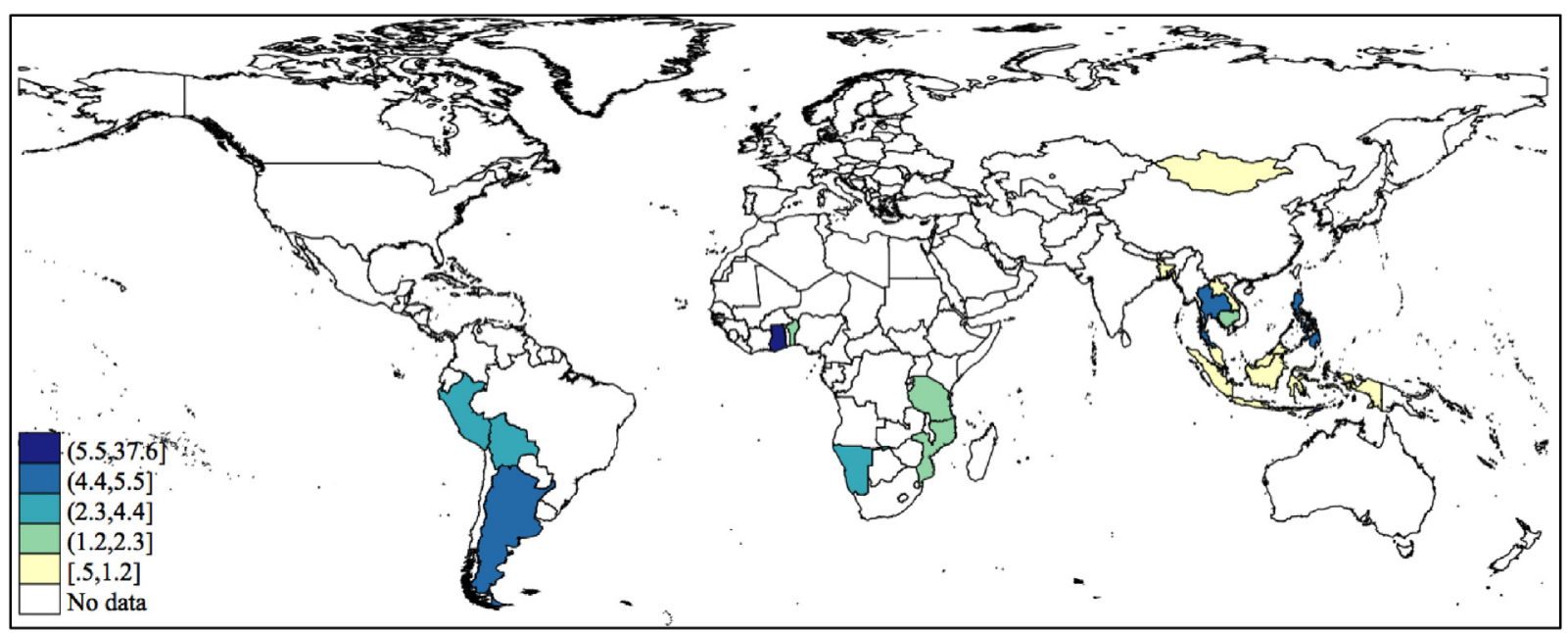

(b) Lifetime cannabis use

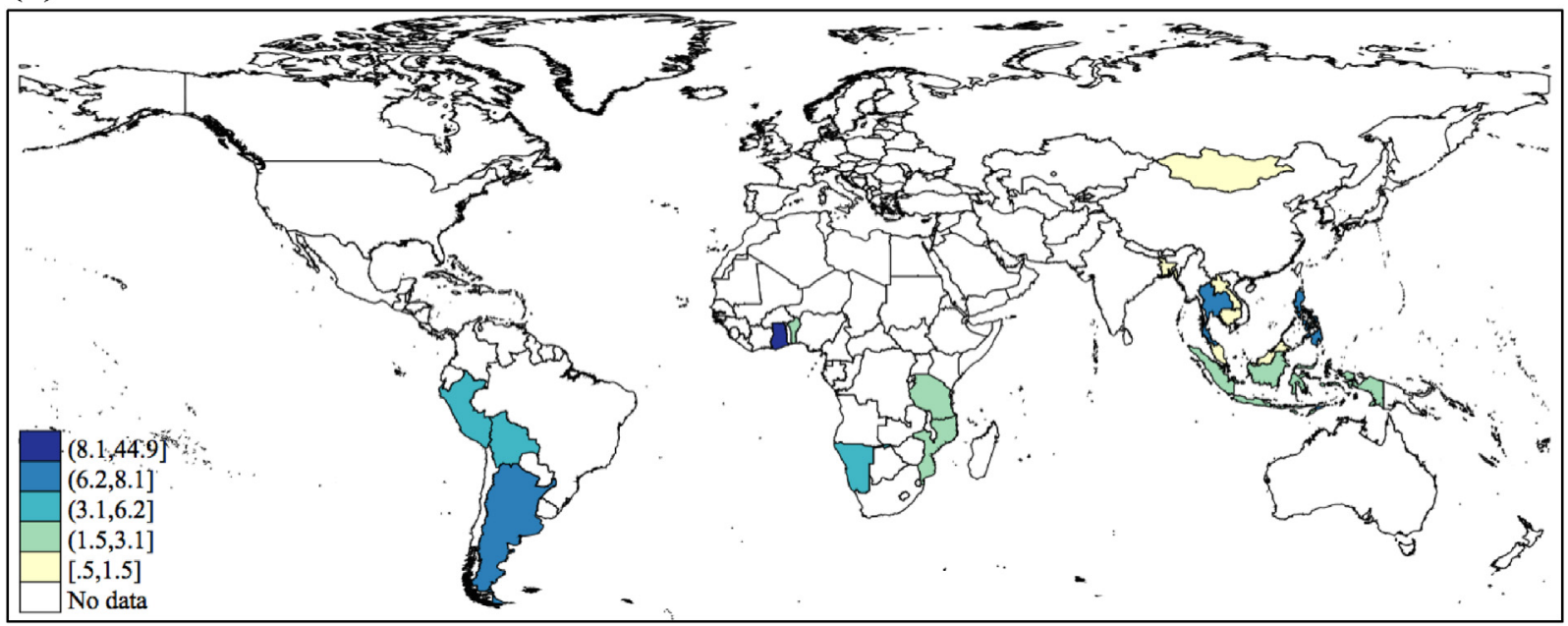

Fig. 1. Age and sex-adjusted prevalence of (a) past 30-day and (b) lifetime cannabis use.

consumption, amphetamine use, smoking, and anxiety-induced insomnia in the model attenuated the OR with the largest attenuation observed with amphetamine use followed by smoking. After adjustment for all potential confounders, past 30-day cannabis use was associated with a $2.03(95 \% \mathrm{CI}=1.42-2.91)$ times higher odds for suicide attempts. The results for lifetime cannabis use were similar with the OR $(95 \% \mathrm{CI})$ of the fully adjusted model being 2.30 (1.73-3.04). There were no significant interactions by sex in the association between cannabis use and suicide attempts.

\section{Discussion}

Although both cannabis use and suicide attempts are highly prevalent among adolescents in our samples, some geographical variations are worth mentioning. This is consistent with evidence that points to the high burden attributed to suicide and substance use disorders in youths living both in upper-income countries and in LMICs [15]. Our findings also suggest that any cannabis use during the past 30 days or during lifetime are associated with a

Table 3

Association between cannabis use and suicide attempts estimated by multivariable logistic regression.

\begin{tabular}{|c|c|c|c|c|}
\hline \multirow[b]{2}{*}{ Models } & \multicolumn{4}{|c|}{ Cannabis use } \\
\hline & \multicolumn{2}{|c|}{ Past 30 days } & \multicolumn{2}{|c|}{ Lifetime } \\
\hline Base model $^{\mathrm{a}}$ & $9.64^{*}$ & {$[7.36,12.61]$} & $9.26^{*}$ & {$[7.23,11.84]$} \\
\hline Base model + alcohol consumption & $5.64^{*}$ & {$[4.35,7.32]$} & $5.54^{*}$ & {$[4.42,6.94]$} \\
\hline Base model + amphetamine use & $3.46^{*}$ & {$[2.53,4.71]$} & $4.62^{*}$ & {$[3.53,6.06]$} \\
\hline Base model + smoking & $5.03^{*}$ & {$[3.75,6.75]$} & $5.14^{*}$ & {$[3.97,6.67]$} \\
\hline Base model + anxiety-induced insomnia & $8.96^{*}$ & {$[6.60,12.17]$} & $8.65^{*}$ & {$[6.55,11.42]$} \\
\hline Fully adjusted model ${ }^{\mathrm{b}}$ & $2.03^{*}$ & {$[1.42,2.91]$} & $2.30^{*}$ & {$[1.74,3.04]$} \\
\hline
\end{tabular}

Data are odds ratio [95\% confidence interval].

a Base model is adjusted for sociodemographic factors (i.e., age, sex, food insecurity, country).

b Fully adjusted model is adjusted for age, sex, food insecurity, country, alcohol consumption, amphetamine use, smoking, and anxiety-induced insomnia.

" $\mathrm{P}<0.001$. 
greater likelihood of suicide attempts. Furthermore, these associations remained robust and significant after adjustment for relevant confounders including the use of other substances and socio-demographic variables. Those results are in accordance to previous school-based reports $[9,10,16]$. However, previous studies were predominantly conducted in high-income countries and there is a lack of multinational studies of this kind [2], with the exception of the survey conducted by Kokkevi et al. [10] which included a school-based sample derived from 16 European countries, and found that any cannabis use in the past 30 days was significantly associated with suicide attempts among adolescents. However, after adjusting to potential confounding variables the magnitude of the association ( $\mathrm{OR}=1.47 ; 95 \% \mathrm{CI}$ : $1.18-1.58)$ [10] appeared somewhat smaller than the one observed in this study $(\mathrm{OR}=2.03 ; 95 \% \mathrm{CI}: 1.42-2.91)$. The current study fills a gap in the literature as it examines the association of cannabis use and suicide attempts in a multinational sample of adolescents attending schools across 21 LMICs.

The associations between cannabis use and suicide attempts were attenuated after adjustment to alcohol use and particularly after multivariable adjustment to smoking and amphetamine consumption. Those findings are consistent with previous studies and reviews that indicate that the use of alcohol or other drugs may decrease the magnitude of the association between cannabis use and suicidality $[2,10,17,18]$.

Delta-9-tetrahidrocannabinol (THC) and cannabidiol (CBD) are the two main ingredients in cannabis [19]. THC is thought to be the main responsible for the addictive properties of cannabis and the resulting subjective euphoria, anxiety/dysphoria, and cognitive effects. On the other hand, CBD has anxiolytic effects and may also mitigate some psychoactive effects related to THC although there are some discrepancies across studies $[19,20]$. Nevertheless, differences in the relative concentrations of THC and CBD in different strains of cannabis may contribute to the associations herein reported, and hence may also partly explain differences observed across geographic regions.

\subsection{Strengths and limitations}

The main strengths of the current study include the enrollment of the largest sample of adolescents $(86,254)$ to date on this topic and its multi-national scope. Most research that had previously examined this association derives from Western countries [2,3], while this study provides data from many LMICs. However, our findings should be interpreted in light of some limitations. First, as with many other school-based surveys, this is a cross-sectional study, and hence the direction of causality cannot be established. Second, evidence indicates that chronic cannabis use may be associated with the development of depressive disorders [21]. Evidence suggests that anxiety and insomnia are strongly related to depression in adolescents [22,23]. We adjusted our findings for the presence of anxiety-induced insomnia, which to a certain degree may thus proxy the presence of depression. Consistently, anxiety-induced insomnia was also associated with suicide attempts across samples from all examined geographical regions. This finding is consistent with previous evidence which suggests that sleep disturbances and particularly insomnia could be associated with suicidal behaviors $[24,25]$. However, the lack of control for the presence of depression is a main limitation of the current work. Third, we controlled our findings to the use of other substances (e.g. alcohol). However, the use of alcohol in the past 30 days may not necessarily reflect the presence of problematic drinking or an alcohol use disorder. Fourth, although food insecurity is closely related to socioeconomic status $[26,27]$, a more comprehensive measure of socioeconomic status could have been more appropriate. Finally, our findings are based on self-reported data. Nevertheless, it has been demonstrated that anonymous self-report questionnaires may elicit more valid data on sensitive issues compared to other methods in this age group [28].

\subsection{Implications}

The strong and independent associations between cannabis use and suicide attempts in a large sample of adolescents from 21 LMICs herein reported open relevant research and clinical implications. First, the causality of this association warrant confirmation in subsequent prospective and Mendelian Randomization studies [29]. Our findings suggest that cannabis use should be taken into consideration when assessing suicide risk in adolescents particularly those living in LMICs. In addition, the current findings may inform preventative public health interventions targeted at adolescents atrisk for suicide residing in LMICs.

\subsection{Conclusions}

In conclusion, our findings point to a significant association between cannabis use and suicidal attempts among adolescents attending schools in disparate world regions. Cannabis use should be taken into account in the prevention and assessment of suicidal behaviors in adolescence. For example, universal, school-based interventions may be effective for the prevention of risk behaviors including cannabis use among the youth [30]. Furthermore, these potential risks should be taken into account during the ongoing international debates around legalization of recreational cannabis use. However, the causality of the association herein reported warrants confirmation in large-scale prospective and Mendelian randomization studies.

\section{Funding}

AK's work was supported by the Miguel Servet contract financed by the CP13/00150 and PI15/00862 projects, integrated into the National R + D + I and funded by the ISCIII - General Branch Evaluation and Promotion of Health Research - and the European Regional Development Fund (ERDF-FEDER). BS is supported by Health Education England and the National Institute for Health Research ICA Programme Clinical Lectureship (ICA-CL-2017-03001). The views expressed in this publication are those of the author(s) and not necessarily those of the NHS, the National Institute for Health Research or the Department of Health and Social Care.

\section{Availability of data and materials}

The datasets generated during and/or analyzed during the current study are available in the Global school-based Student Health Survey repository, available at: http://www.who.int/ncds/ surveillance/gshs/country/en/.

\section{Authors' contributions}

AFC and AK wrote the first draft of the manuscript. AK conducted data analysis. All authors have contributed to the interpretation of the data and have provided meaningful intellectual contribution. All authors read and approved the manuscript prior to submission.

\section{Ethics approval and consent to participate}

Ethical approval was obtained from ethical boards at each study site. Participants give written informed consent. Details are available at: http://www.who.int/ncds/surveillance/gshs/country/en/. 


\section{Consent for publication}

Not applicable.

\section{Competing interests}

The authors declare they have no competing interests.

\section{Appendix A. Supplementary data}

\author{
Supplementary material related to this article can be found, in \\ the online version, at doi:https://doi.org/10.1016/j. \\ eurpsy.2018.10.006.
}

\section{References}

[1] Ferrari A.J., Norman RE, Freedman G, Baxter AJ, Pirkis JE, Harris MG, et al. The burden attributable to mental and substance use disorders as risk factors for suicide: findings from the Global Burden of Disease Study. PLoS One 2010;2014 (9)e91936.

[2] Borges G, Bagge CL, Orozco R. A literature review and meta-analyses of cannabis use and suicidality. J Affect Disord 2016;195:63-74.

[3] Breet E, Goldstone D, Bantjes J. Substance use and suicidal ideation and behaviour in low- and middle-income countries: a systematic review. BMC Public Health 2018:18:549.

[4] Iemmi V, Bantjes J, Coast E, Channer K, Leone T, McDaid D, et al. Suicide and poverty in low-income and middle-income countries: a systematic review. Lancet Psychiatry 2016;3:774-83.

[5] Jacob KS. Suicide prevention in low- and middle-income countries: part perceptions, partial solutions. Br J Psychiatry 2017;211:264-5.

[6] Lubman DI, Cheetham A, Yucel M. Cannabis and adolescent brain development. Pharmacol Ther 2015;148:1-16.

[7] Renard J, Rushlow WJ, Laviolette SR. Effects of adolescent THC exposure on the prefrontal GABAergic system: implications for schizophrenia-related psychopathology. Front Psychiatry 2018;9:281.

[8] Chadwick B, Miller ML, Hurd YL. Cannabis use during adolescent development: susceptibility to psychiatric illness. Front Psychiatry 2013;4:129.

[9] Borges G, Benjet C, Orozco R, Medina-Mora ME, Menendez D. Alcohol, cannabis and other drugs and subsequent suicide ideation and attempt among young Mexicans. J Psychiatr Res 2017;91:74-82.

[10] Kokkevi A, Richardson C, Olszewski D, Matias J, Monshouwer K, Bjarnason T. Multiple substance use and self-reported suicide attempts by adolescents in 16 European countries. Eur Child Adolesc Psychiatry 2012;21:443-50.

[11] Silins E, Horwood LJ, Patton GC, Fergusson DM, Olsson CA, Hutchinson DM, et al. Young adult sequelae of adolescent cannabis use: an integrative analysis. Lancet Psychiatry 2014;1:286-93.

[12] Clarke MC, Coughlan H, Harley M, Connor D, Power E, Lynch F, et al. The impact of adolescent cannabis use, mood disorder and lack of education on attempted suicide in young adulthood. World Psychiatry 2014;13:322-3.
[13] Brener ND, Collins JL, Kann L, Warren CW, Williams BI. Reliability of the youth risk behavior survey questionnaire. Am J Epidemiol 1995;141:575-80.

[14] Vancampfort D, Stubbs B, Firth J, Van Damme T, Koyanagi A. Sedentary behavior and depressive symptoms among 67,077 adolescents aged 12-15 years from 30 low- and middle-income countries. Int J Behav Nutr Phys Act 2018;15:73.

[15] Erskine HE, Moffitt TE, Copeland WE, Costello EJ, Ferrari AJ, Patton G, et al. A heavy burden on young minds: the global burden of mental and substance use disorders in children and youth. Psychol Med 2015;45:1551-63.

[16] Zarrouq B, Bendaou B, Elkinany S, Rammouz I, Aalouane R, Lyoussi B, et al. Suicidal behaviors among Moroccan school students: prevalence and association with socio-demographic characteristics and psychoactive substances use: a cross-sectional study. BMC Psychiatry 2015;15:284.

[17] Degenhardt L, Ferrari AJ, Calabria B, Hall WD, Norman RE, McGrath J, et al. The global epidemiology and contribution of cannabis use and dependence to the global burden of disease: results from the GBD 2010 study. PLoS One 2013;8: e76635.

[18] Degenhardt L, Hall W. Extent of illicit drug use and dependence, and their contribution to the global burden of disease. Lancet (Lond, Engl) 2012;379:5570.

[19] Boggs DL, Nguyen JD, Morgenson D, Taffe MA. Clinical and preclinical evidence for functional interactions of cannabidiol and delta(9)-tetrahydrocannabinol. Neuropsychopharmacology 2018;43:142-54.

[20] Rong C, Lee Y, Carmona NE, Cha DS, Ragguett RM, Rosenblat JD, et al. Cannabidiol in medical marijuana: research vistas and potential opportunities. Pharmacol Res 2017;121:213-8.

[21] Lev-Ran S, Roerecke M, Le Foll B, George TP, McKenzie K, Rehm J. The association between cannabis use and depression: a systematic review and meta-analysis of longitudinal studies. Psychol Med 2014;44:797-810.

[22] Roberts RE, Duong HT. Depression and insomnia among adolescents: a prospective perspective. J Affect Disord 2013;148:66-71.

[23] Johnson EO, Roth T, Breslau N. The association of insomnia with anxiety disorders and depression: exploration of the direction of risk. J Psychiatr Res 2006;40:700-8.

[24] Pigeon WR, Pinquart M, Conner K. Meta-analysis of sleep disturbance and suicidal thoughts and behaviors. J Clin Psychiatry 2012;73:e1160-7.

[25] Pompili M, Innamorati M, Forte A, Longo L, Mazzetta C, Erbuto D, et al. Insomnia as a predictor of high-lethality suicide attempts. Int J Clin Pract 2013:67:1311-6.

[26] Cook JT, Black M, Chilton M, Cutts D, Ettinger de Cuba S, Heeren TC, et al. Are food insecurity's health impacts underestimated in the U.S. population? Marginal food security also predicts adverse health outcomes in young U.S. children and mothers. Adv Nutr (Bethesda, Md) 2013;4:51-61.

[27] Pai N, Kandasamy S, Uleryk E, Maguire JL. Social risk screening for pediatric inpatients. Clin Pediatr 2016;55:1289-94.

[28] Safer DJ. Self-reported suicide attempts by adolescents. Ann Clin Psychiatry 1997;9:263-9.

[29] Katikireddi SV, Green MJ, Taylor AE. Assessing causal relationships using genetic proxies for exposures: an introduction to Mendelian randomization. Addiction 2018:113:764-74.

[30] MacArthur G, Caldwell DM, Redmore J, Watkins SH, Kipping R, White J, et al. Individual-, family-, and school-level interventions targeting multiple risk behaviours in young people. Cochrane Database Syst Rev 2018;10: Cd009927. 